\title{
Compressive Direction Finding Based on Amplitude Comparison
}

\author{
Ruiming Yang, Yipeng Liu, Qun Wan and Wanlin Yang \\ Department of Electronic Engineering, University of Electronic Science and Technology of China \\ Chengdu, China \\ Emial:\{ shan99, liuyipeng,wanqun,wlyang\}@uestc.edu.cn
}

\begin{abstract}
This paper exploits recent developments in sparse approximation and compressed sensing to efficiently perform the direction finding. The new method is proposed based on unimodal characteristic of antenna pattern and sparse property of received data. Unlike the conventional methods based peak-searching and symmetric constraint, the sparse reconstruction algorithm requires less pulse and takes advantage of compressive sampling. Simulation results validate the performance of the proposed method is better than the conventional methods.
\end{abstract}

Index Terms-direction finding; beam scanning; sparse reconstruction; compressive sampling

\section{INTRODUCTION}

Radar is an electromagnetic system for the detection and location of objects. It operates by transmitting a particular type of waveform, a pulse-modulated sine wave for example, and detects the nature of the echo signal. Radar used to extend the ability of one's senses for observing the environment, especially the sense of vision. The value of radar lies not in being a substitute for the eye, but in doing what the eye cannot do. Radar cannot resolve detail as well as the eye, nor is it capable of recognizing the "color' of objects to the degree of sophistication of which eye is capable. However, radar can be designed to see through those conditions impervious to normal human vision, such as darkness, haze, fog, rain, and snow. In addition, with the development of radar technology and the complication of target background, more and more information which is not range but also angle need be known to target in order to track and orientate accurately. In most modern radar systems, the target direction of arrival is estimated by the monopulse technique [1], which in principle can work with just a single pulse.

Different from the direction-finding methods of monopulse radar, there is another method that works as follows: The beam of radar antenna scans to find the user; then the user responses; finally the radar measures the strength of the response signal, and finds the user's location to the radar by the modulation information of the pattern. As the radar antenna pattern has obvious peak

This work was supported in part by the National Natural Science Foundation of China under grant 60772146, the National High Technology Research and Development Program of China (863 Program) under grant 2008AA12Z306 and in part by Science Foundation of Ministry of Education of China under grant 109139.) features, so the user position relative to the radar can be determined directly using the estimated peak location method.

There are many ways to estimate the peak position. An efficient algorithm for estimating the peak position of a sampled function is the Hilbert Transform interpolation algorithm [2]. The algorithm is a computationally efficient algorithm for the peak detection and position estimation of a signal function. It is based on a signal interpolation technique which relies on the Hilbert Transform of the sampled signal. Besides, another methods such as the multi-resolution method which is able to overcome the sampling period's influence on the peak position estimation accuracy, Fourier transform time shift invariant Methods and Sinc function interpolation method [3] can estimate the peak location too.

This paper re-examines the angle estimation problem and uses recent results in sparse approximation [4] and compressive sensing to provide a fundamentally different direction finding method. First we get a sparse representation of the received signal and then the user's location to radar is obtained by the sparse solution. Comparing with the traditional unimodal characteristic and symmetry constraints based maximum (SCBM) methods, the proposed one requires fewer pulses, is with the ability of compressed sampling, and achieves a much smaller estimation error than the traditional search method.

This paper is organized as follows. The beam scanning background and compressed sensing review are described in Section II and section III. In section IV we presented the measurements model. We introduce four direction finding methods in section $\mathrm{V}$ : the traditional maximum method and symmetry constraints based maximum method, the match pursuit and basis pursuit methods which based on the compressive sensing. Section VI presents simulation results that validate the formulation and demonstrate significant performance increase over traditional maximum methods. Conclusions are presented in section VII

\section{BEAM SCANNING BACKGROUND}

Radio signals broadcast from a single antenna will spread out in all directions, and likewise a single antenna will receive signals equally from all directions. This leaves the radar with the problem of deciding where the target object is located. The narrow, directive beam that is 
characteristic of most radar antennas not only concentrates the energy on target but also permits a measurement of the direction to the target. A typical antenna beamwidth for the detection or tracking of aircraft might be about 1 or $2^{\circ}$ [16]. Because radar antennas typically have directive beams, coverage of wide angular regions requires that the narrow beam be scanned rapidly and repeatedly over that region to assure detection of targets wherever they may appear.

Mechanically steered parabolic reflector antennas and electronically steered phased array antennas both find wide application in radar. For example, a dedicated tracking radar generally has a symmetrical antenna which radiates a pencil-beam pattern. The usual ground-based air surveillance radar that provides the range and azimuth of a target generally uses a mechanically rotated reflector antenna with a fan-shaped beam, narrow in azimuth and broad in elevation. Airborne radars and surface-based 3D air surveillance radars (those that rotate mechanically in azimuth to measure the azimuth angle but use some form of electronic steering or beamforming to obtain the elevation angle) often employ planar array apertures. Mechanical scanning of the radar antenna is usually quite acceptable for the vast majority of radar applications [17].

When it is necessary to scan the beam more quickly than can be achieved with mechanical scanning and when high cost can be tolerated, the electronically steered phased array antenna can be employed. (Beam steering with electronically steered phased arrays can be accomplished in microseconds or less if necessary). The electronically scanning offers advantages over mechanically scanned antennas such as instantaneous beam scanning, the availability of multiple concurrent agile beams, and concurrently operating radar modes. This is often used in a phased array radar.

The phase array radar uses an array of similar aerials suitably spaced, the phase of the signal to each individual aerial being controlled so that the signal is reinforced in the desired direction and cancels in other directions. If the individual aerials are in one plane and the signal is fed to each aerial in phase with all others then the signal will reinforce in a direction perpendicular to that plane. By altering the relative phase of the signal fed to each aerial the direction of the beam can be moved because the direction of constructive interference will move. Because phased array radars require no physical movement the beam can scan at thousands of degrees per second, fast enough to irradiate and track many individual targets, and still run a wide-ranging search periodically. By simply turning some of the antennas on or off, the beam can be spread for searching, narrowed for tracking, or even split into two or more virtual radars. However, the beam cannot be effectively steered at small angles to the plane of the array, so for full coverage multiple arrays are required, typically disposed on the faces of a triangular pyramid.

Phased array radars have been in use since the earliest years of radar use in World War II, but limitations of the electronics led to fairly poor accuracy. Phased array radars were originally used for missile defense. They are the heart of the ship-borne Aegis combat system, and the Patriot Missile System, and are increasingly used in other areas because the lack of moving parts makes them more reliable, and sometimes permits a much larger effective antenna, useful in fighter aircraft applications that offer only confined space for mechanical scanning.

As the price of electronics has fallen, phased array radars have become more and more common. Almost all modern military radar systems are based on phased arrays, where the small additional cost is far offset by the improved reliability of a system with no moving parts. Traditional moving-antenna designs are still widely used in roles where cost is a significant factor such as air traffic surveillance, weather radars and similar systems.

Phased array radars are also valued for use in aircraft, since they can track multiple targets. The first aircraft to use a phased array radar is the B-1B Lancer. The first aircraft fighter to use phased array radar was the Mikoyan MiG-31. The MiG-31M's SBI-16 Zaslon phased array radar is considered to be the world's most powerful fighter radar. Phased-array interferometry or, aperture synthesis techniques, using an array of separate dishes that are phased into a single effective aperture, are not typically used for radar applications, although they are widely used in radio astronomy. Because of the Thinned array curse, such arrays of multiple apertures, when used in transmitters, result in narrow beams at the expense of reducing the total power transmitted to the target. In principle, such techniques used could increase the spatial resolution, but the lower power means that this is generally not effective. Aperture synthesis by postprocessing of motion data from a single moving source, on the other hand, is widely used in space and airborne radar systems [18].

\section{COMPRESSED SENSING REVIEW}

Compressive Sensing (CS) [5-7] theory asserts that one can recover certain signals from far fewer samples or measurements than traditional methods use, such as natural images or communications signals, have a representation in terms of a sparsity inducing basis (or sparsity basis for short) where most of the coefficients are zero or small and only a few are large. For example, smooth signals and piecewise smooth signals are sparse in a Fourier and wavelet basis, respectively. Without losing generality, considering a signal $\mathbf{x}$ can be expanded in an orthogonal complete dictionary, with the representation as:

$$
\mathbf{x}_{N \times 1}=\boldsymbol{\Psi}_{N \times N} \mathbf{b}_{N \times 1}
$$

when most elements of the vector $\mathbf{b}$ are zeros, the signal $\mathbf{x}$ is sparse. And when the number of nonzero elements of $\mathbf{b}$ is $\mathrm{S}$ ( $\mathrm{S}<<\mathrm{L}<\mathrm{N})$, the signal is said to be $S$-sparse. Compressed Sensing (CS) provides an alternative to the well-known Shannon sampling theory. It is a framework performing non-adaptive measurement of the informative part of the signal directly on condition that the signal is sparse or compressible.

In compressive sensing, instead of measure the signal 
directly as Nyquist sampling, a $M \times N$ measurement matrix $\Phi$ is used to sample the signal. In matrix notation, the obtained random sample vector can be represented as

$$
\mathbf{y}_{M \times 1}=\boldsymbol{\Phi}_{M \times N} \mathbf{x}_{N \times 1},
$$

Where $M=O(S \log (N / S)) \ll N$.

To reconstructing the signal from $\mathbf{y}$, we need to determine the sparsest signal that explains the measurement $\mathbf{y}$. The strictest measure of sparsity is the $\ell_{0}$ pseudonorm of the signal, define as the number of the nonzero coefficients of the signal. Unfortunately, the $\ell_{0}$ pseudonorm is combinatorial complex to optimize for; there is a NP-hard problem. Compressive sensing enforces sparsity by $\ell_{1}$ norm instead, which is defined as $\|\mathbf{x}\|_{1}=\sum_{i}\left|x_{i}\right|$. In some cases, including the classical compressive sensing reconstruction methods, minimizing the $\ell_{1}$ norm has been theoretically proven equivalent to minimizing the $\ell_{0}$ pseudonorm of the signal.

The quality of the reconstruction depends on the compressibility of the signal, the choice of the reconstruction algorithm, and the incoherence of the sampling dictionary with the sparsity basis. The coherence between the measurement matrix $\boldsymbol{\Phi}$ and the representation basis $\boldsymbol{\Psi}$ is

$$
\mu(\boldsymbol{\Phi}, \boldsymbol{\Psi})=\sqrt{N} \cdot \max _{1 \leq k, j \leq N}\left|\left\langle\varphi_{k}, \psi_{j}\right\rangle\right|
$$

The coherence measures the largest correlation between any two columns of $\boldsymbol{\Phi}$ and $\boldsymbol{\Psi}$.If $\boldsymbol{\Phi}$ and $\boldsymbol{\Psi}$ contain correlated elements, the coherence is large. Otherwise, it is small. Its value obeys that $\mu(\boldsymbol{\Phi}, \boldsymbol{\Psi}) \in[1, \sqrt{2}]$. CS is mainly concerned with low coherence pairs. There are three examples of such pairs. The first is that $\boldsymbol{\Phi}$ is the canonical or spike basis $\varphi_{k}(t)=\delta(t-k)$ and $\boldsymbol{\Psi}$ is the Fourier basis, $\psi_{j}(t)=n^{-1 / 2} e^{i 2 \pi j t / N}$. The time-frequency obeys $\mu(\boldsymbol{\Phi}, \boldsymbol{\Psi})=1$ and we have the maximal incoherence. The second example takes wavelets bases for $\boldsymbol{\Psi}$ and noiselets for $\boldsymbol{\Phi}$. The last example is the most useful. Since the randomly generated dictionaries are universal in the sense that, with very high probability, they are incoherent with any fixed sparsity basis. This property makes such dictionaries very desirable for compressive sensing application.

A sufficient but not necessary condition on the measurement matrix $\boldsymbol{\Phi}$ to recovery the signal is a restricted isometry property (RIP). This property provides a guarantee on the performance of $\Phi$ in CS. It can be stated as:

$$
\left(1-\delta_{s}\right)\|\mathbf{x}\|_{2}^{2} \leq\|\mathbf{\Phi} \mathbf{x}\|_{2}^{2} \leq\left(1+\delta_{s}\right)\|\mathbf{x}\|_{2}^{2}
$$

for all $S$-sparse $\mathbf{x}$. The restricted isometry constant $\delta_{s} \in(0,1)$ is defined as the smallest constant for which this property holds for all S-sparse vectors $\mathbf{x}$.
There are three kinds of frequently used measurement matrices:

1) Non-Uniform Subsampling (NUS) or Random Subsampling matrices which are generated by choosing $M$ separate rows uniformly at random from the unit matrix $\mathbf{I}_{\mathrm{N}}$;

2) Matrices formed by sampling the i.i.d. entries $(\boldsymbol{\Phi})_{\mathrm{ij}}$ from a white Gaussian distribution;

3) Matrices formed by sampling the i.i.d. entries $(\boldsymbol{\Phi})_{\mathrm{ij}}$ from a symmetric Bernoulli distribution and the elements are $\pm 1 / \sqrt{N}$ with probability $1 / 2$ each.

When the RIP holds, a series of recovering algorithm can reconstruct the sparse signal [8]. One is greedy algorithm, such as matched pursuit (MP) [9], OMP [10]; another group is convex programming, such as basis pursuit (BP)[19], LASSO [20] and Dantzig Selector (DS)[11]. DS has almost the same performance as LASSO. Both of the convex programming and greedy algorithm have advantages and disadvantages when applied to different problem scenarios. A very extensive literature has been developed that covers various modifications of both algorithms so to emphasize their strengths and neutralize their flaws. A short assessment of their differences would be that convex programming algorithm has a more reconstruction accuracy while greedy algorithm has less computing complex. And in contrast to BP, LASSO has additional denoising performance advantage.

\section{MEASUREMENT MODEL}

As described in section I, the radar antenna pattern has obvious peak features, so the user position relative to the radar can be determined directly using the estimated peak location method. Assume that the antenna pattern is $p(\theta)$. Without loss of generality, as shown in Figure 1, let $p(\theta)$ be a Sinc function and represented as:

$$
p(\theta)=\operatorname{sinc}^{2}\left(\theta_{b}^{-1} \theta\right)
$$

where $\theta_{b}$ is the half of the mainbeam width. In the $k$-th moment, the strength of the received signal can be represented as:

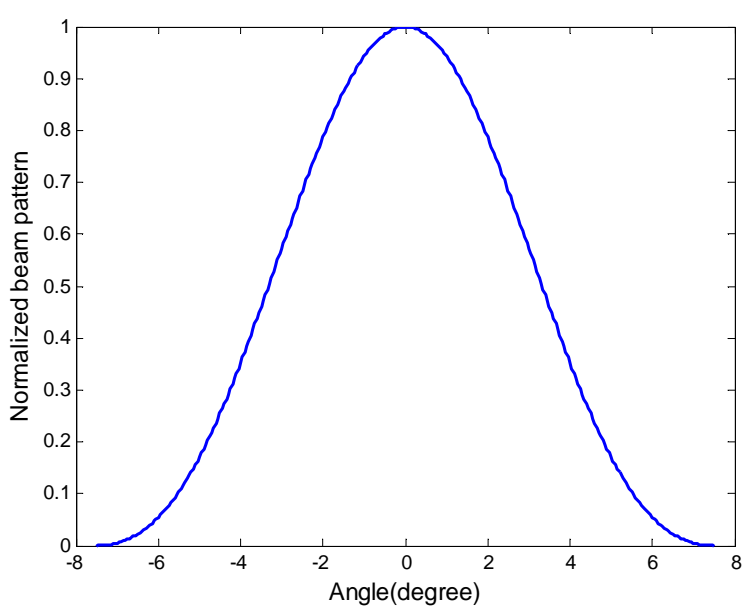

Fig. 1 the normalized beam pattern of radar antenna 


$$
x_{k}=s_{k}+v_{k}
$$

where $s_{k}=p\left(\theta_{k}\right) ; \theta_{k}$ is the radar antenna scanning angle in the $k$-th moment; $v_{k}$ is the noise, which is in $\chi^{2}$ distribution, $k=1,2, \cdots, K, K$ is the number of received signal strength in the measurement period.

In (2), $s_{k}$ has only the information of the received signal strength along with the change of radar antenna's scanning angle. Thus $v_{k}$ is already normalized by the maximum received signal strength.

\section{DIRECTION FINDING BEAM}

\section{A.Maximum Method}

Maximum method takes advantage of peak characteristic of the pattern $p(\theta)$. The user's relative azimuth to the radar is estimated by finding the location corresponding to the maximum element in the received signal's strength sequences $x_{1}, x_{2}, \cdots, x_{K}$, i.e.

$$
\hat{\theta}_{\mathrm{MAX}}=\theta_{\hat{k}},
$$

where

$$
\hat{k}=\arg \max _{1 \leq k \leq K}\left(x_{k}\right)
$$

\section{B. Symmetry Constraints Based Maximum Method}

The symmetry constraints based maximum (SCBM) method uses both the unimodal characteristic and the symmetry. It estimates the user's azimuth relative to the radar by finding the best symmetrical location corresponding to the maximum element in the user's received signal strength sequences, i.e:

$$
\hat{\theta}_{\text {SYM }}=\theta_{\hat{k}},
$$

where

$$
\hat{k}=\arg \max _{k}\left(\frac{x_{m} \sum_{m=1}^{N} x_{k-m} x_{k+m}}{\sqrt{\sum_{m=1}^{N} x_{k-m}^{2} \sum_{m=1}^{N} x_{k+m}^{2}}}\right),
$$

$\mathrm{N}$ is the minimum element between $k-1$ and $K-k$, i.e.

$$
N=\min (k-1, K-k),
$$

\section{Match Pursuit}

Searching over an extremely large dictionary for the best matches is computationally unacceptable for practical applications. Mallat and Zhang proposed a greedy solution that is known from that time as Matching Pursuit. Matching pursuit is a type of numerical technique which involves finding the "best matching" projections of multidimensional data onto an over-complete dictionary. The basic idea is to represent a signal from Hilbert space as a weighted sum of functions (called atoms). By taking an extremely redundant dictionary we can look in it for functions that best match a signal. Finding a representation where most of the coefficients in the sum are close to 0 (sparse representation) is desirable for signal coding and compression.

Here we divide the beam width into $2 L+1$ sections with the same length, i.e. $\theta_{-L}, \theta_{-L+1}, \ldots, \theta_{k}, \ldots, \theta_{L}$, where $\theta_{k}=\theta_{b} \cdot k / L, \quad k=-L,-L+1, \cdots, 0,1, \cdots, L$, and construct the Redundant dictionary as :

$$
\mathbf{D}=\operatorname{Toeplitz}\left(\mathbf{d}_{1}, \mathbf{d}_{2}\right)
$$

where

$$
\begin{gathered}
\mathbf{d}_{1}=\left[\begin{array}{llllllll}
p\left(\theta_{0}\right) & p\left(\theta_{1}\right) & \cdots & p\left(\theta_{L}\right) & 0 & 0 & \cdots & 0
\end{array}\right]^{T}(13) \\
\mathbf{d}_{2}=\left[\begin{array}{llllllll}
p\left(\theta_{0}\right) & p\left(\theta_{-1}\right) & \cdots & p\left(\theta_{-L}\right) & 0 & 0 & \cdots & 0
\end{array}\right]
\end{gathered}
$$

where $p\left(\theta_{k}\right)=\operatorname{sinc}^{2}\left(\theta_{b}^{-1} \theta_{k}\right)$, and the column vector $D_{i}(i=1, \ldots, 2 L+1)$ of the redundant dictionary is named as atom.

$$
\mathbf{x}=\mathbf{D s}+\mathbf{v}
$$

where $\mathbf{x}=\left[x_{1}, \ldots, x_{K}\right]^{T}, \quad \mathbf{v}=\left[v_{1}, \ldots, v_{K}\right]^{T}$. The user's azimuth to the radar can be determined by finding the non-zero element of vector $\mathbf{s}$, and the corresponding atom can be obtained by finding the maximum correlation of the atom and received signal $\mathbf{x}$, i.e.[12]:

$$
D_{o p t}=\arg \max _{i}\left|\left\langle D_{i}, x\right\rangle\right|,
$$

\section{Basis Pursuit}

To encourage sparsity, The $\ell_{0}$ optimization is optimal but non-convex and known to be NP-hard. In practice, a multitude of efficient algorithms have been proposed, which achieve high recovery rates. The $\ell_{1}$-minimization method is the most extensively studied recovery technique. In this approach, the non-convex $\ell_{0}$ norm is replaced by the convex $\ell_{1}$ norm. This approximation is known as Basis Pursuit (BP) which is a principle for decomposing a signal into an "optimal" superposition of dictionary elements, where optimal means having the smallest $\ell_{1}$ norm of coefficients among all such decompositions.

Here the sparse solution of (11) can be obtained by optimization method. It can be modeled as:

$$
\min \|\mathbf{s}\|_{1}+\sum_{i=1}^{K} e_{i} \quad \text { s.t. } \quad e_{i} \geq 0, \quad s_{i} \geq 0,
$$

where $\mathbf{e}=\mathbf{x}$ - Ds . It is obviously that (10) is a convex programming and the solution can be obtained by some optimization software, such as the software cvx [13]. 


\section{SIMULATION EXPERIMENT}

In this section we present simulation results that demonstrate the performance of our method. For the remainder of this section we suppose that in the radar's beam scans process, the number of the responded impulse is 31 ; and the changes in beam scanning angle corresponding to the adjacent pulse interval is 0.1 degrees. The radar pattern is $p(\theta)=\operatorname{sinc}^{2}\left(\theta_{b}^{-1} \theta\right)$,

$\theta_{b}=7.5^{\circ}$. We did 1000 independent experiments count the error probability of the angle finding.

Fig. 2 to Fig. 6 demonstrate the performance of the four methods introduced in section $\mathrm{V}$, when the data extraction rates are one-half, one-quarter, one-sixth, oneeigthth and one-tenth respectively. The results of our basis pursuit algorithm are marked by pentacle and labeled 'BP'. While the ones of match pursuit, SCBM method, and the traditional maximum method are marked by diamond, rectangle and circularity, respectively. They are respectively labeled by 'MP', 'SCBM' and 'MAX'. The table 1 gives the corresponding mean square error of direction estimation by different methods.

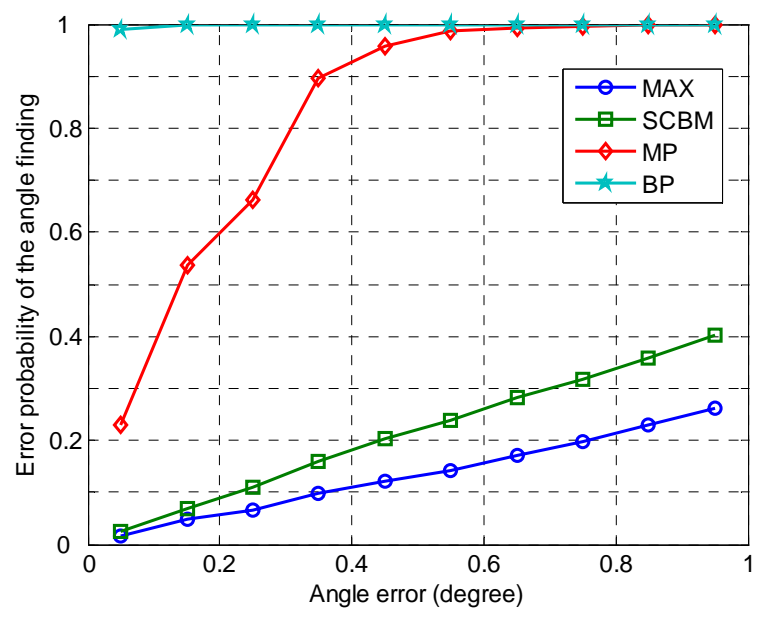

Fig. 2 Standard deviation of the direction error (extraction rate: one-half, $\mathrm{SNR}=5 \mathrm{~dB}$ )

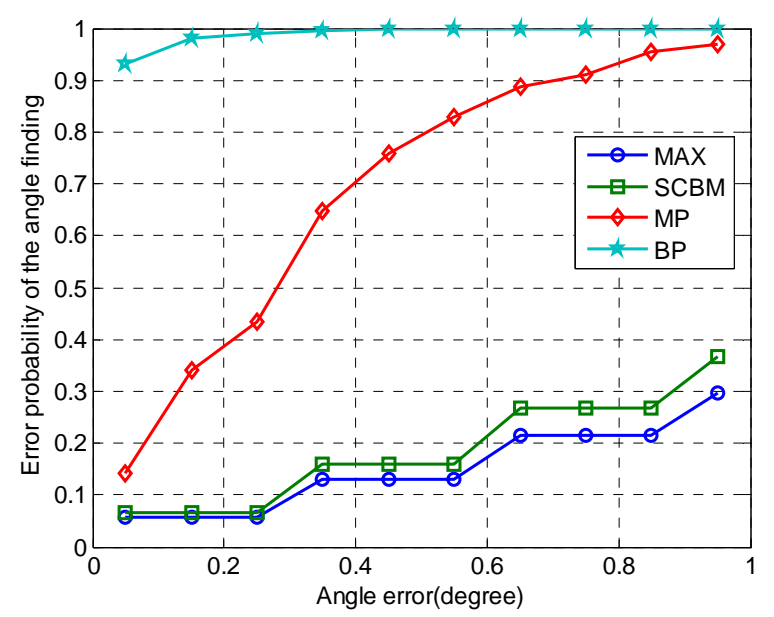

Fig.4 Standard deviation of the direction error (extraction rate: one-sixth, $\mathrm{SNR}=5 \mathrm{~dB}$ )
The simulation results above show that the estimates based on sparse signal representation are better than the traditional maximum method and SCBM method. The proposed method can give a more accurate result even the volume of data is relatively few. Matching pursuit method can get the user's azimuth to the radar only by calculating the correlation between the atom and received signal. It is simple and easy to implement. However, due to influence of the correlation between adjacent atoms in the redundant dictionary, the performance of the match pursuit is inferior to standard basis pursuit method. The basis pursuit can optimize the optimal atom to get the solution, and the performance is much superior to others. However, compared with the matching pursuit method, the base basis pursuit method is more complex.

\section{CONCLUSION}

Our results demonstrate that the angle finding can be significant improved if we incorporate the sparse information processing method into the radar antenna pattern modulated direction finding. The experimental results show that sparse signal representation based

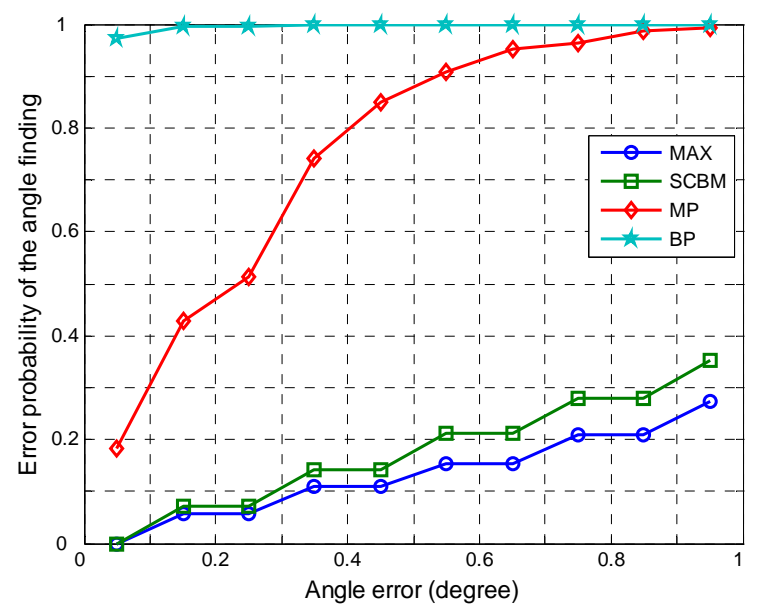

Fig. 3 Standard deviation of the direction error (extraction rate: one-quarter, $\mathrm{SNR}=5 \mathrm{~dB}$ )

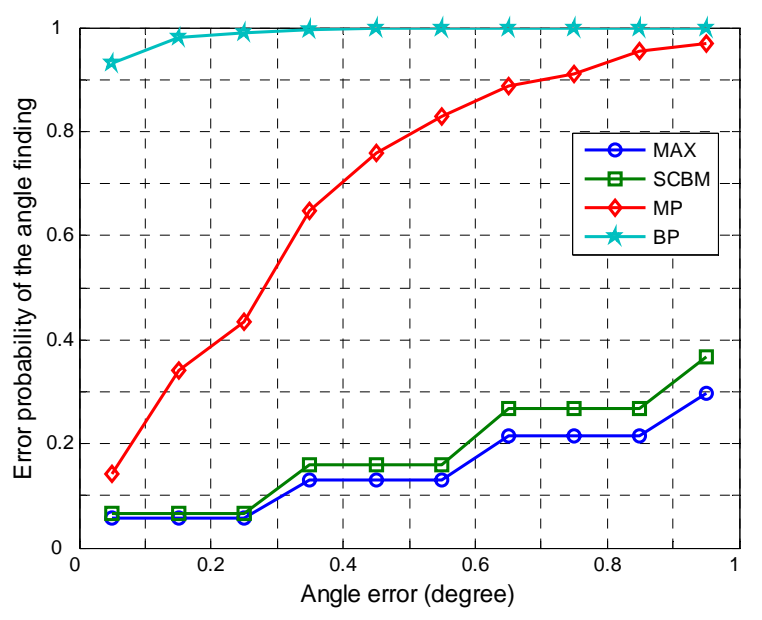

Fig. 5 Standard deviation of the direction error (extraction rate: one-eight, $\mathrm{SNR}=5 \mathrm{~dB}$ ) 
TABLE I. MEAN Square ERror of Direction ESTIMATION (DEGREE)

\begin{tabular}{|c|c|c|c|c|}
\hline $\begin{array}{c}\text { Sample's } \\
\text { extraction rate }\end{array}$ & Maximum method & SCBM method & MP & BP \\
\hline One-half & 3.2045 & 2.3244 & 0.2356 & 0.0152 \\
\hline One-quarter & 3.2231 & 3.0339 & 0.3385 & 0.0282 \\
\hline One-sixth & 3.1453 & 3.1607 & 0.4282 & 0.0545 \\
\hline One-eight & 3.1389 & 3.1224 & 0.4842 & 0.0762 \\
\hline One-tenth & 3.1493 & 3.2983 & 0.5294 & 0.0937 \\
\hline
\end{tabular}

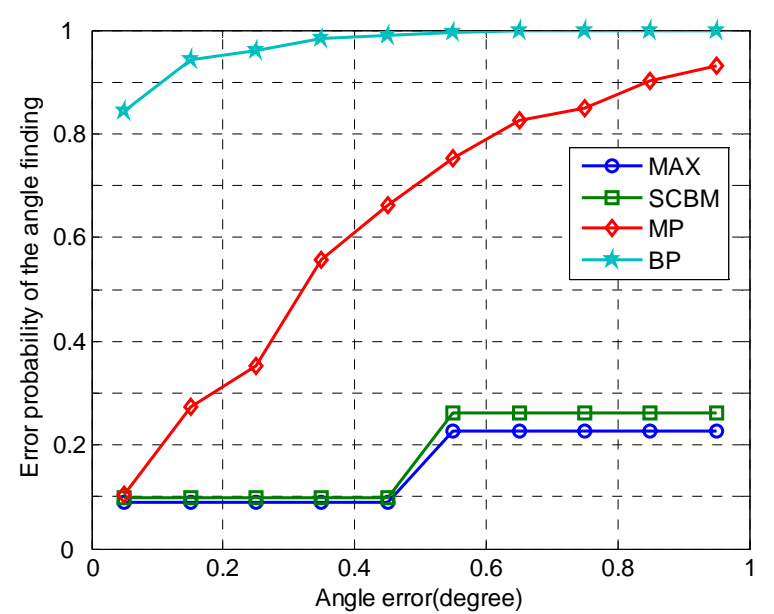

Fig.6 Standard deviation of the direction error (extraction rate: one-tenth, $\mathrm{SNR}=5 \mathrm{~dB}$ )

estimation is better than the traditional maximum method and the SCBM method. Although the base basis pursuit method is more computational complex in contrast to the matching pursuit method, the performance of the BP algorithm is much superior to the MP method for as much as the influence of the correlation between adjacent atoms in the redundant dictionary.

\section{ACKNOWLEDGMENT}

The authors thank anonymous reviewers for their valuable suggestions.

\section{REFERENCES}

[1] Skolnik, M., "Introduction to Radar Systems," New York: McGraw-Hill, 2001.

[2] S. S. Abeysekera, "An efficient Hilbert transform interpolation algorithm for peak position estimation,"Proceedings of the 11th IEEE Signal Processing Workshop on Statistical Signal Processing, 6-8 Aug. 2001, pp.417- 420

[3] Chu-Xiong Ding, Jing Bai, "Peak position estimation algorithms for cross-correlation function in elastography,"Proceedings of the 20th Annual International Conference of the IEEE Engineering in Medicine and Biology Society, 29 Oct.-1 Nov. 1998, pp. $866-868$

[4] A. C. Gilbert, J. A. Tropp. "Applications of sparse approximations in communications," in Proc. of IEEE Int. Symp. Inf. Theory, 2005.

[5] Candes, E.J.; Wakin, M.B. "An introduction to compressive sampling", IEEE Signal Processing Magazine, Volume 25, Issue 2, pp. 21-30, March 2008
[6] D. Donoho. Compressed sensing. IEEE Trans. Inform. Theory, Vol. 52, No. 4: 1289-1306, Apr. 2006.

[7] E. Candès. Compressive sampling. in Proceedings of Int. Congress of Mathematics, Madrid, Spain, 2006.

[8] E.J.Candes. "The restricted isometry property and its implication for compressive sensing," C.R.Math. Acad. Sci. Paris, Seris I, 346:589, 2008

[9] S. G. Mallat and Z. Zhang.: 'Matching pursuits with timefrequency dictionaries', IEEE Tran. on ASSP, Dec. 1993, vol. 41, no. 12, pp. 3397-3415

[10] Tropp J , Gilbert A. "Signal recovery from random measurements via orthogonal matching Pursuit", Transacting on Information Theory, 2007, 53(12):46554666.

[11] Emmanuel Candes, and Terence Tao, "The Dantzig selector: statistical estimation when $\mathrm{p}$ is much larger than n”, Annals of Statistics 2007. Volume 35, Number 6, pp. 2313-2351

[12] Yipeng Liu, Qun Wan, Xiaoli Chu, "A Robust Beamformer Based on Weighted Sparse Constraint" CoRR abs/1005.4200, 2010, [online] accessiable: http://arxiv.org/abs/1005.4200

[13] M. Grant and S. Boyd , CVX: Matlab Software for Disciplined Convex Programming. Online accessiable : http://stanford.edu/ boyd/cvx

[14] Ying Zhang, Qun Wan, Anmin Huang, "Localization of narrow band source in the present of mutual coupling via sparse solution finding, "Progress in Electromagnetics Research-PIER, 2008, vol.86, pp.243-257

[15] Yipeng Liu, Qun Wan, "Sparse Support Recovery with Phase-Only Measurements" CoRR abs/1005.1801, 2010, [online] accessiable: http://arxiv.org/abs/1005.1801

[16] M.I.Snolnik. Radar Handbook, Second Edition, New York: McGraw-Hill Publishing Company, 1980.

[17] Peyton Z, Peables Radars Principles, John wiley and Sons Inc, 1998.

[18] H.Griffiths, A Tutorial on Synetheic Aperture Radar. IEEE National Conferrance, 1997.

[19] Deanna Needell, Topics in Compressed Sensing, Submitted in partial satisfaction of the requirements for the degree of doctor of philosophy in mathematics in the office of graduate studies of the university of California DAVIS.

[20] D. Omidiran and M. J. Wainwright. High-dimensional subset recovery in noise: Sparsified measurements without loss of statistical efficiency. Technical report, Department of Statistics,UC Berkeley, April 2008. Short version presented at Int. Symp. Info. Theory, July 2008. 


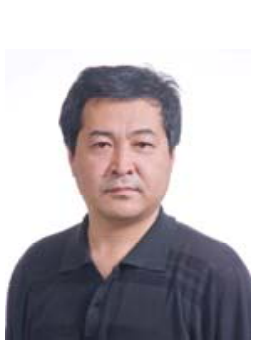

Ruiming Yang was born in Shandong. $\mathrm{He}$ received his $\mathrm{M}$. S from Beijing institute of technology. Now he is a postgraduate with the Electronic Engineering (EE) department, UESTC, Chengdu, China.

His general research interest focuses on array signal processing for radar system, mobile localization.

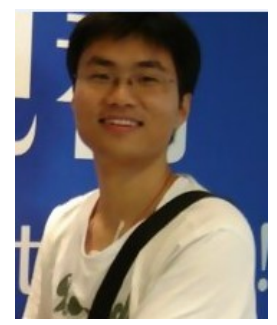

Yipeng Liu received the B. Eng. degree in biomedical engineering (BME) from University of Electronic Science and Technology of China (UESTC), Chengdu, China, in 2006. Since 2006, he has been a postgraduate with the Electronic Engineering (EE) department, UESTC, Chengdu, China.

His general research interest focuses on signal processing for wireless communication and reconnaissance.

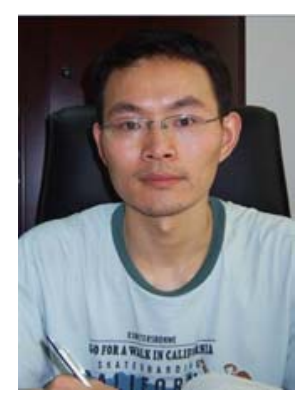

Qun Wan received the B.S. degree from Nanjing University in 1993, the M.S. degree from UESTC in 1996, and the Ph.D. degree from UESTC in 2001.

During 2001-2002, he was a postdoctor at Tsinghua University, where he participated in cellular localization program. In 2003, he was a Technical Staff at UTstarcom. Since 2004, he has been a Professor in the Department of Electronic Engineering at the University of Electronic Science and Technology of China (UESTC). His research interests include sparse and array signal processing, mobile and indoor localization. $\mathrm{He}$ is a Senior Member of CIE.

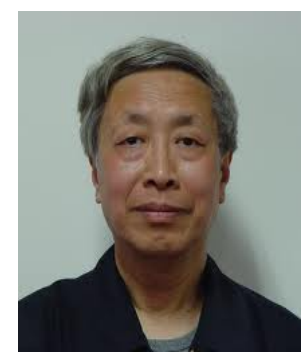

Wanlin Yang received the B. Eng. and $\mathrm{M} . \mathrm{S}$ degree in electronic engineering from University of Electronic Science and Technology of China (UESTC), Chengdu, China in 1967, and 1981, respectively, and the M. S in electronic engineering from University of Pennsylvania in 1985.

He was an associated professor in the Department of Electronic Engineering at the University of Electronic Science and Technology of China (UESTC) from 1987 to 1992. Since 1992, he has been a professor in UESTC. He is the head of the Radar System Lab of Department of Electronic Engineering. His research interests include microwave imaging, radar system, array signal processing, etc. 